\title{
PROBLEMS ASSOCIATED WITH THE USE OF EXTERNAL URINARY COLLECTORS IN THE MALE PARAPLEGIC
}

\author{
By John B. Nanninga, M.D. and Joel Rosen, M.D. \\ Spinal Cord Injury Centre Northwestern Memorial Hospital and \\ Rehabilitation Institute of Chicago, Chicago, Illinois 606II, U.S.A.
}

USE of an external collecting apparatus for male spinal cord injury patients has become more prevalent in our experience as more of these patients are subjected to intermittent catheterisation and perhaps subsequent external sphincterotomy or TUR of the bladder neck. Also, elderly males with uninhibited type of neurogenic bladder often find an external urinal helpful in preventing bed wetting. Beneficial though these external urinary collecting systems may be, there are problems associated with their use. Among these are inflammation of glans and prepuce, skin excoriation, skin necrosis and ulceration, and urethral fistula formation. This communication seeks to demonstrate problems associated with external collectors which we have encountered and offer an opinion as to treatment and prevention.

External collectors for the male patient may be categorised into two general types (Pearman \& Shah, 1973). First there is the type consisting of a drainage sheath attached to a binder or supporting apparatus. This apparatus usually fastens around the waist and thighs and the penis is inserted into the sheath. To prevent leakage, the proximal end of the sheath must be firmly set against the skin at the base of the penis, and herein lies the problem. Too much pressure, or eccentrically placed pressure on one side of the junction of the sheath to the binder, can result in skin breakdown. Figure I demonstrates the erosion of the
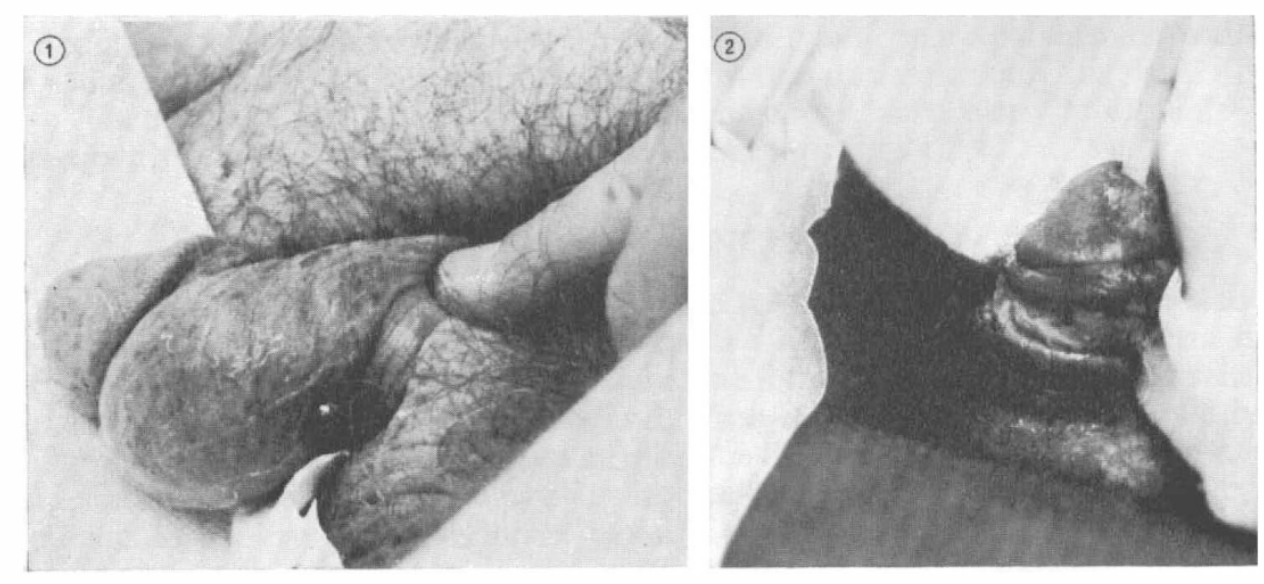

FIG. I

Constricting ring at the base of penis with distal edema.

FIG. 2

Concentric erosion of penile skin resulting from a constricting band of tape. 


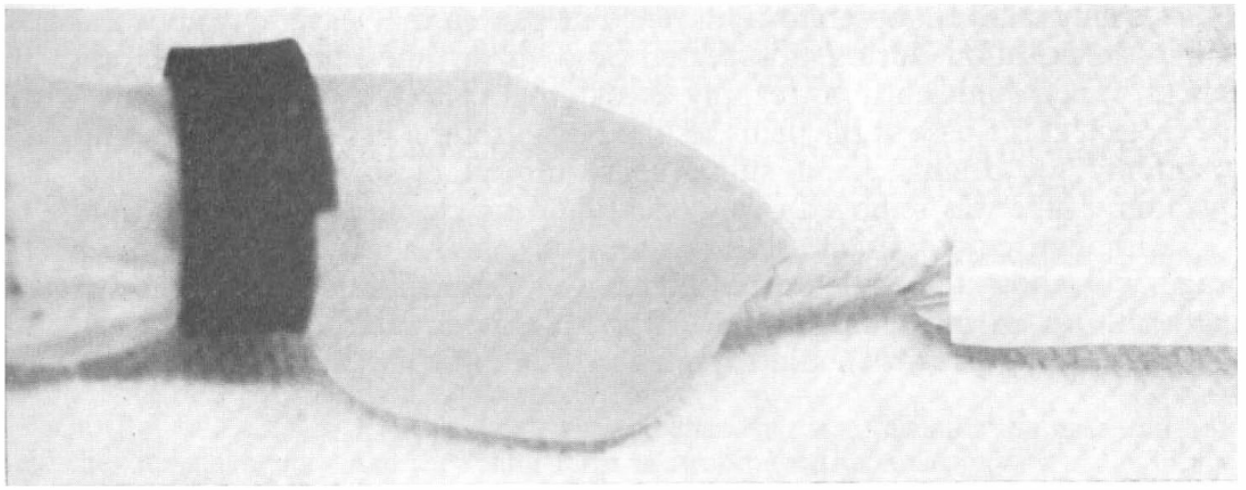

FIG. 3

Stasis of urine caused by twisting of distal end of external collector.

skin at the penoscrotal junction resulting from the aforementioned type of collector. In another patient, extensive erosion at the base of the penis resulted in a urethrocutaneous fistula. Another disadvantage of this type of collector is that adjustment of the sheath and supporter is difficult and, in the tetraplegic patient, requires additional aid from one or two persons. Even simple body movements can cause a shift in pressure at the base of the penis. Our service does not use this type of apparatus because of the difficulties of applying and adjusting it and because of the risk of skin breakdown.

The other type of external collector consists of a thin latex or plastic condom applied to the penis and secured either by elastic tape applied circumferentially proximal to the glans penis or by some form of skin adhesive applied to the penis. The sheath is changed every one to three days. The latex is thin and translucent enough so that cyanosis, indicating impaired venous drainage, and lesions of the penile skin should be apparent to the patient. As might be anticipated, the application of tape under too much tension can lead to skin breakdown (fig. 2). With the resulting ulceration, urine may leak between the collector and skin causing further irritation to adjacent skin.

In some uncircumcised patients, the prepuce may become macerated because of the continued contact of urine. A twist or kink in the drainage tube or at the end of the collector itself contributes to the stasis of urine at the distal end of the penis (fig. 3). This problem of twisting may be obviated by placing two or three strips of tape longitudinally from the external collector to the connecting tubing. Also a semirigid adaptor may be placed at the distal end of the external to prevent the latex from twisting (Pearman \& Shah, 1973). The treatment of the inflammatory response on the skin usually requires removal of the external collector to allow for drying and healing. Drying lotions may help promote healing if there is an exudate. Patients in whom the prepuce becomes inflamed and excoriated may require circumcision, which also allows the patient to tape the external collector securely in place just proximal to the glans. We have, on occasion, recommended circumcision for patients in whom the external collector continually slipped off.

For the ulcerated or necrotic lesion, surgical debridement may be necessary. We have not used skin grafting but simply allowed the area to heal by granulation. 
This usually takes about ten to I 4 days. The patient may have to resume intermittent catheterisation during this period or wear an indwelling catheter until the skin is healed sufficiently to reapply an external collector. The patient in whom the erosion at the base of the penis resulted in a urethral fistula required suprapubic cystotomy for drainage and subsequent surgical closure of the fistula. The cystotomy tube was removed two weeks following closure of the fistula.

Although external collecting devices can cause a variety of lesions, proper usage will allow the patient the advantage of remaining dry. It is certainly preferable to an indwelling catheter. With proper instruction and warning as the hazards, the patient should experience little difficulty.

\section{REFERENCES}

Pearman, J. W. \& Shah, S. K. (I973). A new adaptor which obviates problems associated with condom external urinary drainage of male patients. Int. F. Paraplegia, II, 25-29.

\section{Discussion}

Chairman: Mr. J. Cosbie-Ross. I should just like to say before opening the discussion on these two papers that I should have thought that a plastic Foley catheter with an infected urine and possibly urea splitters in the urine that calcification is not inevitable but very likely, and I am sure that the Foley catheter is responsible for a tremendous lot of calculi in the bladder and shouldn't be used really, I don't think, in this day and age.

DR. H. TALBOT (U.S.A.). I should like first to ask Dr. Lindan: are those calculi bladder or renal or both? Regarding condom drainage, a couple of precautions are worth emphasis, repeating what Dr. Nanninga just said. A tape or a strap isn't necessary, if the patient or his attendants put it on carefully the use of facing cement of the type used with an ileostomy, if firmly put on with a band of cement perhaps two or two and a half $\mathrm{cm}$. wide it will hold very well because the tubing can be secured on the thigh so that there is no pull on the catheter. Therefore, the cement isn't made to hold the condom against pull but just to make it waterproof. As far as changing is concerned, I think that the best plan is to change it twice a day. I deplore leaving it on longer than that, and we had an eleven and one rule-that it was on II hours, taken off the penis, dried, left there for I hour and then re-applied. A paraplegic can do this easily himself. A tetraplegic needs help either from his family or from attendants, and this takes a little time and a little money but it does ensure a good result, and you don't get anything for nothing.

PROF. AsColi (Italy). Regarding the interesting paper of Dr. Lindan, I have some practical considerations. We have long discussed of what is better, intermittent catheterisation or indwelling catheter. There are tenacious colleagues here who are supporters of the first and others who are supporters of the second method. In my opinion everything depends on the possibility in the end of the medical environment, namely of the condition of the medical staff, of the number and quality of physicians and nurses with excellent urological education and above all of a staff whom you can absolutely trust regarding sterility. The non-touch technique of catheterisation of Sir Ludwig Guttmann is an excellent method, and where I can, I use it. But in hospitals and units where the staff is scarce and where only a few are in a condition of carrying out a proper intermittent catheterisation, the system of the indwelling catheter with or without tidal drainage is preferable. One must not forget the danger of lesions of the urethra which 